Proceedings

\title{
Loop Antenna Driven Double Microwave Resonator- Based Sensors Incorporating PDMS Microchannels on Glass Substrates ${ }^{+}$
}

\author{
Berk Camli 1,*, Emre Altinagac ${ }^{2}$, Huseyin Kizil ${ }^{3}$, Hamdi Torun ${ }^{4}$, Gunhan Dundar ${ }^{1}$ and \\ Arda D. Yalcinkaya ${ }^{1,5}$ \\ 1 Electrical and Electronics Engineering, Bogazici University, Istanbul 34342, Turkey; \\ dundar@boun.edu.tr (G.D.); arda.yalcinkaya@boun.edu.tr (A.D.Y.) \\ 2 Nanoscience and Nanoengineering, Istanbul Technical University, Istanbul 34469, Turkey; \\ emrealtinagac@gmail.com \\ 3 Metallurgical and Materials Engineering, Istanbul Technical University, Istanbul 34469, Turkey; \\ kizilh@itu.edu.tr \\ 4 Mathematics, Physics and Electrical Engineering, Northumbria University, Newcastle NE1 8ST, UK; \\ hamdi.torun@boun.edu.tr \\ 5 Center for Life Sciences and Technologies, Bogazici University, Istanbul 34342, Turkey \\ * Correspondence: berk.camli@boun.edu.tr; Tel.: +90-212-359-7368 \\ † Presented at the Eurosensors 2018 Conference, Graz, Austria, 9-12 September 2018.
}

Published: 16 November 2018

\begin{abstract}
Microwave resonator-based sensors offer low-cost, contactless, label-free sensing solutions in a variety of applications. Sensing is done by the observation of the shifts in resonant frequency of the sensor structure, which depends on resonator geometry, material and physical properties of the environment. It is observed that the readings can be significantly affected by changes in secondary physical parameters or sample localization on resonator. A double microwave resonator sensing system incorporating microchannels on glass substrates are proposed to address these challenges. PDMS microchannels bonded on glass substrates are mounted on split ring resonators fabricated via low-cost processes. Experiments are performed with glucose solutions of $1.4 \mathrm{mg} / \mathrm{mL}-$ $3.0 \mathrm{mg} / \mathrm{mL}$ concentration range. Results confirm that the use of double resonators increase rejection of background noise, whereas microchannel use increases measurement stability. Overall measurement sensitivity is shown to be $0.92 \mathrm{MHz} /(\mathrm{mg} / \mathrm{mL})$. Further improvements are aimed with the bonding of microchannels directly on resonators fabricated on glass substrates.
\end{abstract}

Keywords: split ring resonators; microchannel; PDMS; glucose sensing

\section{Introduction}

There has been a recent interest in the implementation of microwave split ring resonators (SRR) in sensing applications. These are planar conductor loops with slits of them, with different varieties studied in the literature [1]. Geometry of these shapes give raise to inductance and capacitances, which form RLC circuits together, forming passive electromagnetic filters with specific resonance frequencies $\left(f_{0}\right)$. For the simplest SRR formed of a single slit ring, total capacitance $C$ and inductance $L$ determine $f_{0}$ as follows [2]:

$$
f_{0}=\frac{1}{2 \pi \sqrt{L C}}
$$


Since $f_{0}$ of these resonators are related to structure geometry, material composition and the physical characteristics of the environment, it is possible for these them to operate as transducers. Sensing mechanism is based on the fact that a change in $f_{0}$ is an indication of a change in environment. Actuation and read-out can be done via the use of electromagnetic waves, which has positive implications on sensor invasiveness and speed. SRRs also have smaller volumes compared to other similar resonators available [3]. Due to these advantages, SRRs are proposed as low-cost, contactless, label-free and potentially CMOS integrable sensors in a variety of applications $[4,5]$.

Sensing performance of these sensors can be improved when the response to fluctuations due to secondary physical characteristics, such as temperature etc., can be rejected. Additionally, the sample volume and its localization on the resonator surface should be controlled precisely since $f_{0}$ tracking is sensitive to changes in these. A double measurement-based system relying on real-time comparison of one measuring and one reference resonator was demonstrated previously to address the former issue [6].

In this work, we expand the double measurement approach by incorporating PDMS microchannels bonded on glass substrates to the system to control sample volume and localization on sensor surface. The microchannel structures are placed on top of the resonators so that the channels transverse the sensitive gap region. A pair of identical resonators is present, each of which are actuated by a surrounding loop antenna. The system components are shown in Figure 1.
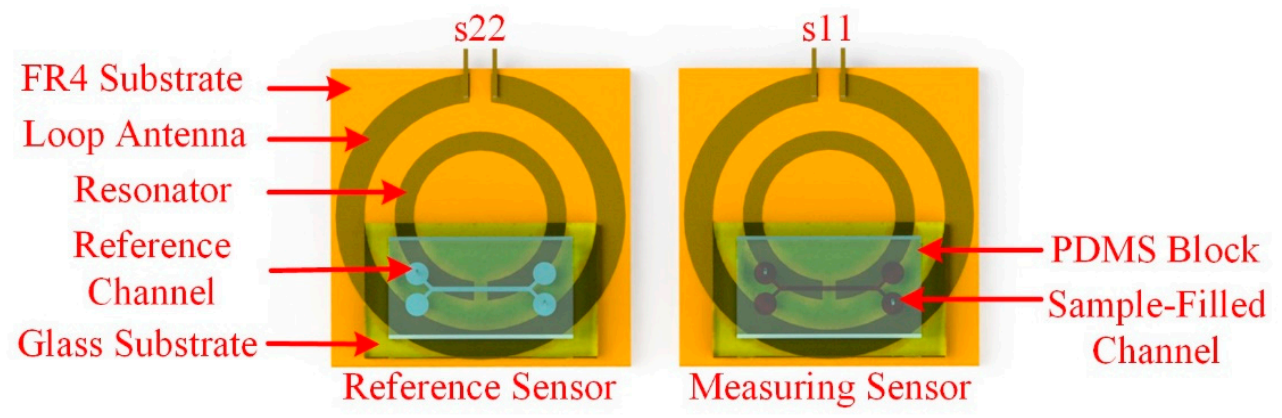

Figure 1. Sensor system used in the experiments.

\section{Results}

The dimensions of a single antenna-resonator couple used in the experiments and simulation results regarding their interactions are shown in Figure 2. Designed dimensions aimed for a $f_{0}$ at 2 $\mathrm{GHz}$, confirmed by the results. Simulations also validated that SRR structures are actuated optimally when they are placed at the center of loop antennas due to increased coupling efficiency.

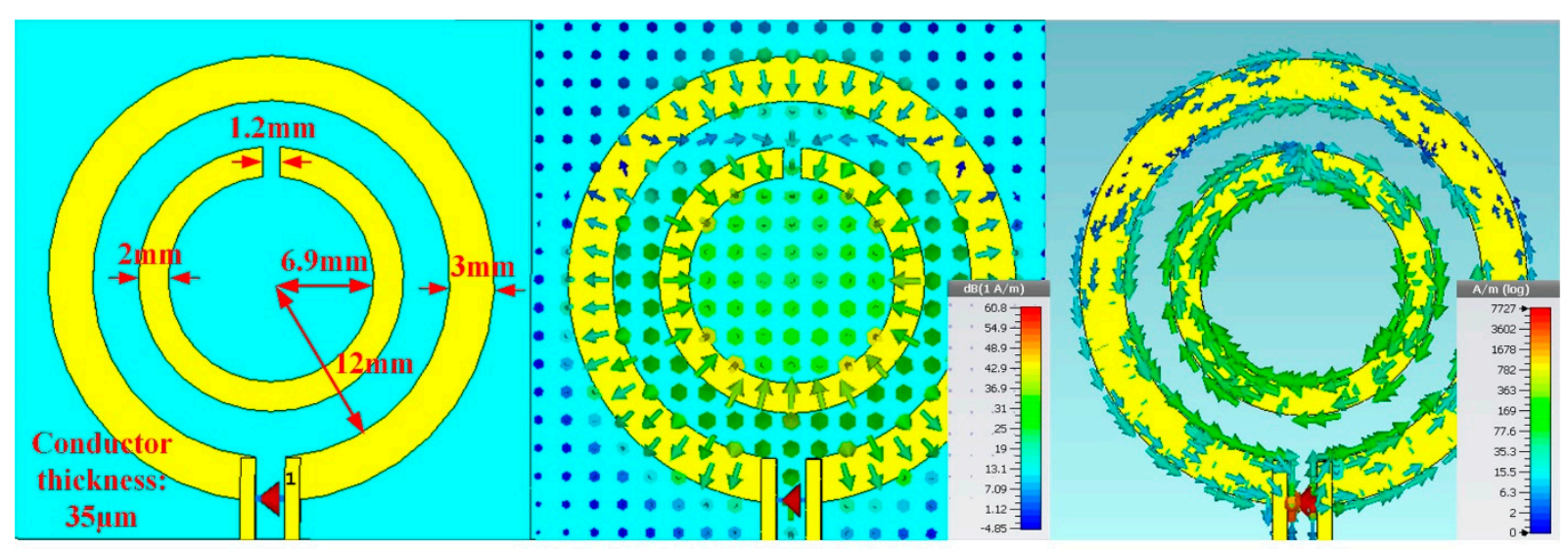

Figure 2. Dimensions and geometry of a resonator-loop antenna couple (left). CST simulation results showing h fields (middle) and surface current density (right). 
Following the sensor fabrication, basic measurements were performed to observe the resonant behavior of the sensors under different circumstances. Here, $f_{0}$ values are de Measured reflection ( $s 11$

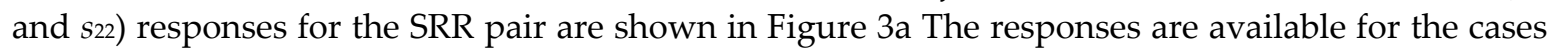
when the SRRs are bare, covered with an empty microchannel each and when the microchannels are filled with DI water. The nominal $f_{0}$ of bare resonators are observed to be in good agreement with design goal of $2 \mathrm{GHz}$. It is seen that $f_{0}$ shifts to lower frequencies as the total dielectric load on the resonator increases with the addition of microchannels and DI water sample. This is expected from Equation (1), since a higher dielectric load produces a higher capacitance of the equivalent $C$ of the system, thus reducing $f_{0}$.

After the basic measurements, experiments were conducted with glucose solution samples of different concentrations in range of $1.4 \mathrm{mg} / \mathrm{mL}-3.0 \mathrm{mg} / \mathrm{mL}$. The measuring resonator was applied glucose concentration, while the reference resonator was kept with an empty channel. Measurements were performed four times per glucose concentration level. Figure $3 b$ shows the average shift of resonant frequency for the measuring and reference resonators and the absolute difference between the two. Error bars demonstrate data range of four trials at each concentration level. Trendline generated from the data $\left(\mathrm{R}^{2}=0.9\right)$ indicate a sensitivity of $0.92 \mathrm{MHz} /(\mathrm{mg} / \mathrm{mL})$.

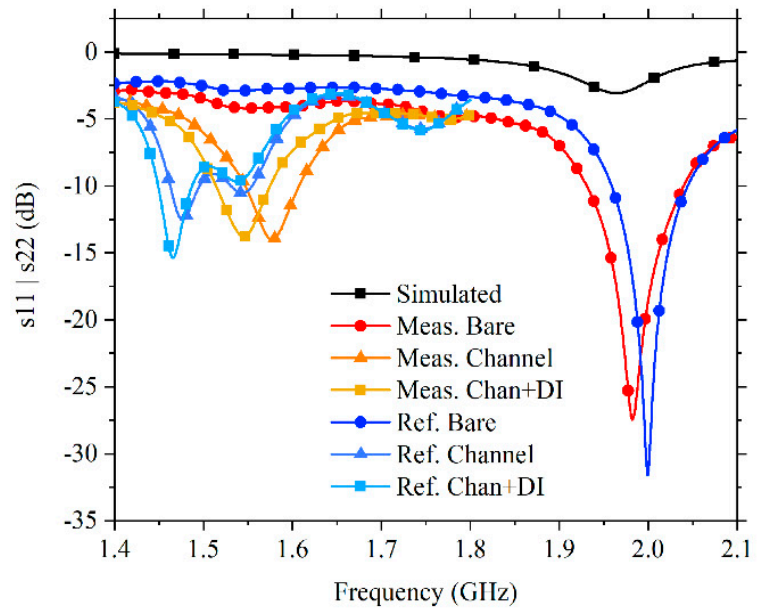

(a)

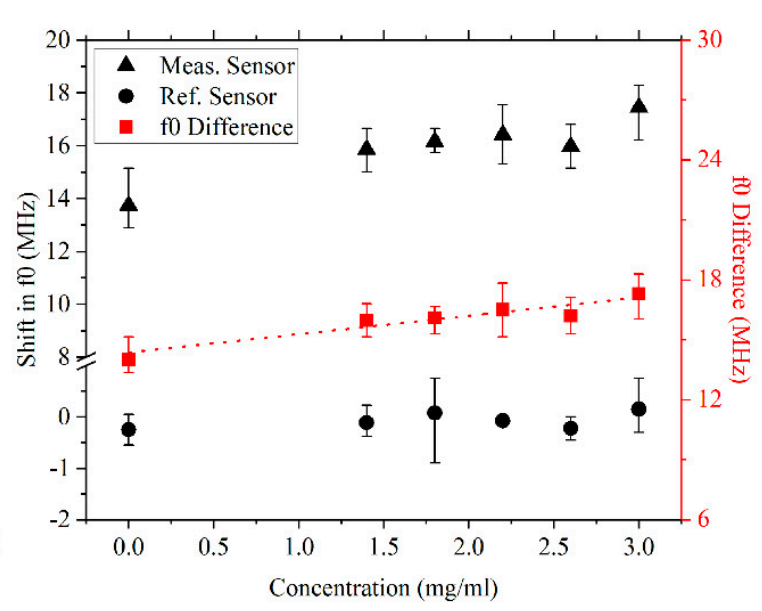

(b)

Figure 3. Reflection responses, measured as $s_{11}$ and $s_{22}$ for bare, empty microchannel added and DI water-loaded microchannel added SRRs (a). Shift amounts in $f_{0}$ for measuring and reference sensors at different glucose concentration levels and the difference of shifts. Trendline fit $\left(R^{2}=0.9\right)$ indicates a sensitivity of $0.92 \mathrm{MHz} /(\mathrm{mg} / \mathrm{mL})(\mathbf{b})$.

\section{Discussion}

In this work loop antennas were used for resonator actuation, in contrast to monopoleantennas used in our previous work [6]. Incorporation of loop antennas surrounding SRRs increases the actuation efficiency reduces the number of ports in the antenna end, while providing clearer reading spectra. The fact that the reference and measuring resonators do not yield the same $f_{0}$ at their bare state is compensated by the fact that the operation does not rely on the ground state of $f_{0}$. It rather follows the shift of $f_{0}$ with respect to this ground state. Arbitrary shift fluctuations read for the reference resonator confirm the presence of noise. The fluctuation pattern is ghosted similarly in te measuring resonator readings. As a result, tracking the difference between the two gives a more linear sensor response with respect to changing glucose concentration levels.

\section{Materials and Methods}

The coupled resonator antenna geometry used in the experiments were designed for a nominal $f_{0}$ of $2 \mathrm{GHz}$. Related simulations were performed in CST Microwave Studio software. Dimensions of 
the determined structure are indicated in Figure 2. This geometry was patterned on $30 \mu \mathrm{m} \mathrm{Cu}$-coated $1.6 \mathrm{~mm}$-thick FR4 substrates via low-cost printed circuit board fabrication techniques. The microchannels were fabricated via PDMS casting. Master molds for casting were created via conventional optical lithography processes, where two layers of SU-8 3050 negative photoresist is spin-coated. The total thickness of the mold achieved this way is $200 \mu \mathrm{m}$. After casting, plasma activated bonding process was applied to PDMS microchannels and $1.1 \mathrm{~mm}$ thick soda lime glass substrates. To achieve bonding, PDMS microchannels and substrates were kept for $15 \mathrm{~min}$ in a vacuum oven heated to $50{ }^{\circ} \mathrm{C}$. PDMS microchannel bonded glass substrates were mounted on resonators so that the microchannel transverses the resonator gap-air interfaces perpendicularly. Fixation of glass substrates on resonators were done via commercially available adhesive agents. DI water and glucose solution of $1.4 \mathrm{mg} / \mathrm{mL}-3.0 \mathrm{mg} / \mathrm{mL}$ concentration range were used as samples, which were applied via syringes with needles. Tracking of $f_{0}$ was done by the observation of reflection parameters with a vector network analyzer (Rohde \& Schwarz ZVB4, Munich, Germany) through SMA connectors soldered to loop antenna ports. Either port of the vector network analyzer can be connected to the pair of resonator structures individually, allowing for simultaneous tracking of the respective $f_{0}$.

Author Contributions: Conceptualization, H.T., G.D. and A.D.Y.; Methodology, B.C. and E.A.; Software, B.C. and E.A.; Validation, B.C., H.T. and A.D.Y.; Formal Analysis, B.C.; Investigation, B.C. and E.A.; Resources, E.A., H.K. and A.D.Y.; Data Curation, B.C.; Writing-Original Draft Preparation, B.C. and E.A.; Writing-Review \& Editing, H.K., H.T., G.D. and A.D.Y.; Visualization, B.C.; Supervision, H.K. and A.D.Y.; Project Administration, A.D.Y.; Funding Acquisition, A.D.Y.

Funding: This research was funded by the Turkish Academy of Sciences (TUBA), the Scientific and Technological Research Council of Turkey (TUBITAK) grant number 112E250, and the Department of Scientific Research of Bogazici University (BAP) grant number 16A02TUG4.

Conflicts of Interest: The authors declare no conflict of interest. The founding sponsors had no role in the design of the study; in the collection, analyses, or interpretation of data; in the writing of the manuscript, and in the decision to publish the results.

\section{References}

1. Baena, J.D.; Bonache, J.; Martin, F.; Sillero, R.M.; Falcone, F.; Lopetegi, T.; Laso, M.A.G.; Garcia-Garcia, J.; Gil, I.; Portillo, M.F.; et al. Equivalent-circuit models for split-ring resonators and complementary split-ring resonators coupled to planar transmission lines. IEEE Trans. Microw. Theory Tech. 2005, 53, 1451-1461. doi:10.1109/TMTT.2005.845211.

2. Sydoruk, O.; Tatartschuk, E.;Shamonina, E.;Solymar, L. Analytical formulation for the resonant frequency of split rings. J. Appl. Phys. 2009, 105. doi:10.1063/1.3056052.

3. Abduljabar, A.A.; Rowe, D.J.; Porch, A.; Barrow, D.A. Novel Microwave Microfluidic Sensor Using a Microstrip Split-Ring Resonator. IEEE Trans. Microw. Theory Tech. 2014, 62, 679-688. doi:10.1109/TMTT.2014.2300066.

4. Camli, B.; Kusakci, E.; Lafci, B.; Salman, S.; Torun, H.; Yalcinkaya, A.D. Cost-Effective, Microstrip Antenna Driven Ring Resonator Microwave Biosensor for Biospecific Detection of Glucose. IEEE J. Sel. Top. Quantum Electron. 2017, 23, 404-409. doi:10.1109/JSTQE.2017.2659226.

5. Lee, H.J.; Yook, J.G. Biosensing using split-ring resonators at microwave regime. Appl. Phys. Lett. 2008, 92. doi:10.1063/1.2946656.

6. Camli, B.; Torun, H.; Dundar, G.; Yalcinkaya, A.D. Reference-Incorporating Microwave Resonator-Based Sensors for Biological Sensing Applications. Proceedings 2017, 1. doi:10.3390/proceedings1040542. 